Pacific Journal of Mathematic 


\section{LIE ALGEBRAS WITH DESCENDING CHAIN CONDITION}

\section{J. Marshall Osborn}

In this note we investigate Lie algebras which satisfy the descending chain condition on ideals of ideals. We show that a Lie algebra $L$ satisfies this descending chain condition if and only if the following two conditions hold: (i) $L$ contains a finite dimensional solvable ideal $N$ such that every solvable ideal of $L$ is contained in $N$, and (ii) $L / N$ is a subdirect sum of a finite number of prime algebras satisfying the descending chain condition. We also show that if $L$ is a prime algebra with this chain condition then there exists a Lie algebra $B$, which is either simple or the tensor product of a simple Lie algebra with a truncated polynomial algebra, such that $L$ is isomorphic to a subalgebra of Der $B$ containing $a d_{B}$.

A decade ago a theory of Jordan algebras with descending chain condition on inner ideals was developed [3, Chapter IV] which emulates and connects with the theory of Artinian rings. More recently Benkart [1] studied Lie algebras with descending chain condition on inner ideals (a subspace $B$ of a Lie algebra $L$ is called an inner ideal of $L$ if $[B,[B, L]] \subseteq B)$. It has not been settled yet whether a Jordan algebra with DCC on inner ideals necessarily has a nilpotent radical. One of the purposes of the present paper is to show that a Lie algebra with DCC on inner ideals has a radical which is solvable and finite dimensional. This follows from the results stated in the last paragraph since any ideal of an ideal is an inner ideal and hence DCC on inner ideals implies DCC on ideals of ideals.

It is known that a finite dimensional semisimple Lie algebra $M$ of characteristic $p$ is not necessarily a direct sum of simple algebras, but there do not seem to be any results published which express $M$ in terms of algebras which belong to a more restricted class than $M$. A second purpose of this paper is to show that $M$ is a subdirect sum of prime algebras. Rather than finite dimensionality the assumption of DCC on ideals of ideals seems to be the most natural level of generality for this proof.

The results in this paper hold for Lie algebras over a field $\Phi$ of any characteristic including 2.

Suppose now that $L$ is a Lie algebra with DCC on ideals of ideals. We begin with

Lemma 1. If $C$ is a solvable ideal of $L$, then $C$ is finite 
dimensional.

Proof. We proceed by induction on the index of solvability of $C$. If $[C, C]=0$, then every subspace of $C$ is an ideal of $C$, and so by DCC we have $\operatorname{dim} C<\infty$. For the inductive step, if $C^{(k)} \neq 0$ and $C^{(k+1)}=\left[C^{(k)}, C^{(k)}\right]=0$, then $C^{(k)}$ is an ideal of $L$ and $\operatorname{dim} C^{(k)}<\infty$ as above. Since the quotient algebra $L / C^{(k)}$ satisfies the same descending chain condition and since $C / C^{(k)}$ is a solvable ideal of $L / C^{(k)}$ of smaller index, it follows from the inductive hypothesis that $\operatorname{dim} C / C^{(k)}<\infty$. Hence $\operatorname{dim} C<\infty$.

We call a Lie algebra semisimple if it contains no nonzero solvable ideals.

LEMMA 2. $L$ contains a finite dimensional solvable ideal $N$ which contains all solvable ideals of $L$, and $L / N$ is semisimple.

Proof. It is sufficient to establish that $L$ contains a maximal solvable ideal $N$, since the uniqueness of $N$ and the semisimplicity of $L / N$ will then follow from the fact that the sum of two solvable ideals is solvable and that the preimage in $L$ of any solvable ideal of $L / N$ is solvable. The finite dimensionality of $N$ will follow from Lemma 1. If $L$ does not contain a maximal solvable ideal, it must contain a properly ascending chain $C_{1} \subset C_{2} \subset C_{3} \subset \cdots$ of solvable ideals of $L$. Each $C_{i}$ is finite dimensional by Lemma 1 . Let $C=\mathrm{U}_{i=1}^{\infty} C_{i}$ and note that $C$ is an infinite dimensional ideal of $L$.

For each positive integer $i$ we define $D_{i}=\left\{d \in C \mid\left[d, C_{i}\right]=0\right\}$, and we note that $D_{i}$ is an ideal of $L$ since it is a subspace and since

$$
\begin{gathered}
{\left[\left[D_{i}, L\right], C_{i}\right] \subseteq\left[\left[D_{i}, C_{i}\right], L\right]+\left[D_{i},\left[L, C_{i}\right]\right]} \\
=\left[D_{i},\left[L, C_{i}\right]\right] \subseteq\left[D_{i}, C_{i}\right]=0
\end{gathered}
$$

Furthermore, $D_{1} \supseteqq D_{2} \supseteq D_{3} \supseteq \cdots$ is a descending sequence of ideals. By the chain condition there must exist an integer $m$ such that $D_{m}=D_{m+i}$ for all positive integers $i$. Then $D_{m}=\bigcap_{i=1}^{\infty} D_{i}$, and we see that $D_{m}$ is the center of $C$. Then every subspace of $D_{m}$ is an ideal of $C$. 'By the descending chain condition, $\operatorname{dim} D_{m}<\infty$.

Consider the map of $C$ onto the ring of endomorphisms of the subspace $C_{m}$ given by $\Phi(c)=\left.a d_{c}\right|_{C_{m}}$. The kernel of $\Phi$ is exactly $D_{m}$. Since $\operatorname{dim} C=\infty$ and $\operatorname{dim} D_{m}<\infty$, the dimension of the image of $\Phi$ must be infinite. But $\operatorname{dim} C_{m}<\infty$ and so the dimension of the ring of endomorphisms of $C_{m}$ is also finite. This contradiction shows that $L$ must have had a maximal solvable ideal, to complete the proof.

LemMa 3. Let $L$ be semisimple and let $B$ be a minimal ideal 
of $L$. Then either $B$ is simple or else $\Phi$ has characteristic $p$ and $B \cong B_{0} \otimes W_{k}$ where $B_{0}$ is a simple Lie algebra and where $W_{k}=$ $\Phi\left[x_{1}, \cdots, x_{k}\right] /\left(x_{1}^{p}, \cdots, x_{k}^{p}\right)$ is the truncated polynomial algebra on $k$ indeterminates.

Proof. If $B$ is a minimal ideal of $L$, then the elements of $a d_{L}$ act on $B$ as derivations and under this action $B$ is derivation simple. Also the chain condition on $L$ implies DCC on the ideals of $B$. Thus $B$ satisfies the hypotheses of Block's theorem [2]. Then the conclusion of Lemma 3 holds since it is just the conclusion of Block's theorem.

If $L$ is semisimple, the sum $S$ of all its minimal ideals will be called the socle of $L$.

LEMMA 4. If $L$ is semisimple, $S$ is a direct sum of the minimal ideals of $L$. Hence $L$ has only a finite number of minimal ideals.

Proof. We construct two sequences of ideals $B_{1}, B_{2}, \cdots$ and $C_{0}, C_{1}, C_{2}, \cdots$ inductively by taking $C_{0}=S$, by choosing each $B_{i}$ for $i \geqq 1$ to be a minimal ideal of $L$ contained in $C_{i-1}$, and by choosing each $C_{i}$ for $i \geqq 1$ to be an ideal of $L$ which is maximal with respect to being contained in $C_{i-1}$ and not containing $B_{i}$. The $C_{i}$ 's form a strictly descending chain of ideals which must stop because of the chain condition. The only way that the process can stop is that $C_{k}=0$ for some integer $k$. If $k$ is the smallest such integer, it is easy to verify that $S$ is a direct sum of $B_{1}, B_{2}, \cdots, B_{k}$.

A Lie algebra will be called prime if it does not contain two nonzero ideals whose product is zero. A prime algebra cannot contain two distinct minimal ideals $B_{1}, B_{2}$, since then $\left[B_{1}, B_{2}\right] \subset B_{1} \cap B_{2}=0$. Thus a prime algebra either contains a unique minimal ideal or no minimal ideals. In the presence of our chain condition, prime algebras must contain a unique minimal ideal. Thus in this paper $L$ is prime only if it contains a unique minimal ideal $B$ such that $\{B, B\rfloor \neq 0$ (and hence $[B, B]=B$ ). Conversely any Lie algebra with a unique minimal ideal $B$ satisfying $[B, B]=B$ is prime, since for any nonzero ideals $C, D$ of $L$ we have $[C, D\rfloor \supseteq\lfloor B, B\rfloor=B \neq 0$.

Lemma 5. If $L$ is semisimple, then $L$ is a subdirect sum of a finite number of prime algebras.

Proof. Let $B_{1}, \cdots, B_{n}$ be the minimal ideals of $L$, and for $1 \leqq$ $i \leqq n$ let $C_{i}$ be an ideal of $L$ which is maximal with respect to not containiag $B_{i}$. Since every ideal $D$ of $L$ properly containing $C_{i}$ must contain $B_{i}$ by the choice of $C_{i}$, we see that every ideal $D / C_{i}$ of $L / C_{i}$ 
must contain $\left(B_{i}+C_{i}\right) / C_{i}$. Thus $\left(B_{i}+C_{i}\right) / C_{i}$ is the unique minimal ideal of $L / C_{i}$. Hence $L / C_{i}$ is prime.

If $\bigcap_{i=1}^{n} C_{i} \neq 0$, then this intersection must contain one of the minimal ideals $B_{j}$ of $L$. But $B_{j} \nsubseteq C_{j}$, so $B_{j} \nsubseteq \bigcap_{i=1}^{n} C_{i}$. Hence $\bigcap_{i=1}^{n} C_{i}=0$. It follows that the homomorphism

$$
L \longrightarrow \sum_{i=1}^{n} \oplus L / C_{i}
$$

defined by composing the natural homomorphisms $L \rightarrow L / C_{i}$ is a faithful representation of $L$ as a subdirect sum of prime algebras.

LeMma 6. If $L$ is prime with minimal ideal $B$, then $L$ is isomorphic to a subalgebra of Der $B$ containing $a d_{B}$.

Proof. Since $a d_{L}$ restricted to $B$ is a subalgebra of Der $B$, the natural $\operatorname{map} \theta:\left.L \rightarrow a d_{L}\right|_{B}$ is a homomorphism of $L$ into Der $B$. Since $[B, B] \neq 0$, the restriction of $\theta$ to $B$ is not zero. Hence the kernel $K$ of $\theta$ is an ideal of $L$ not containing $B$, giving $K=0$. Thus $\theta$ is an isomorphism.

We have proved the forward direction of our

THEOREM. A Lie algebra $L$ satisfies the descending chain condition on ideals of ideals if and only if it satisfies both the conditions

(i) L contains a finite dimensional solvable ideal $N$ such that every solvable ideal of $L$ is contained in $N$,

(ii) $L / N$ is a subdirect sum of a finite number of prime algebras satisfying the descending chain condition.

If $L$ is a prime algebra with descending chain condition on ideals of ideals, then $L$ has a unique minimal ideal $B$ and $L$ is isomorphic to a subalgebra of Der $B$ containing $a d_{B}$. Also, $B$ is either $a$ simple algebra or is the tensor product of a simple algebra with a truncated polynomial algebra.

To show the reverse direction of the first statement of this theorem, let $L$ be Lie algebra satisfying (i) and (ii). Since every ideal of an ideal of $L / N$ is uniquely determined by its images in each of the prime algebras of the subdirect sum, it is easy to see that $L / N$ must also satisfy DCC on ideals of ideals. And then, since $N$ is finite diminsional, $L$ must satisfy the same chain condition itself.

The standard example of a finite dimensional Lie algebra which is semisimple but not a direct sum of simple algebras is constructed as follows. Let $A$ be the Lie algebra of all $p \times p$ matrices over a field of characteristic $p$, and let $Z$ be the center of $A$. Then $A / Z$ 
is semisimple and prime, but not simple. Its unique minimal ideal (and only proper ideal) is the image under $A \rightarrow A / Z$ of the matrices of trace zero in $A$.

This example can be modified to show that a finite dimensional semisimple Lie algebra of characteristic $p$ is not necessarily a direct sum of prime algebras. Consider $2 p$ by $2 p$ matrices which have been partitioned into four $p$ by $p$ blocks, and let $A^{\prime}$ be the Lie algebra of such matrices which have only zeros in their two offdiagonal blocks. If $A^{\prime \prime}$ is the subalgebra of $A^{\prime}$ of matrices in $A^{\prime}$ of trace zero and if $Z^{\prime \prime}$ is the center of $A^{\prime \prime}$, then $A^{\prime \prime} / Z^{\prime \prime}$ is a simisimple Lie algebra which is not a direct sum of prime algebras.

\section{REFERENCES}

1. Georgia M. Benkart, On inner ideals and ad-nilpotent elements of lie algebras, Trans. Amer. Math. Soc.

2. Richard E. Block, Determination of the differentiably simple rings with a minimal ideal, Ann. of Math.. II, vol. 90 (1969), 433-459.

3. Nathan Jacobson, Structure and Representations of Jordan Algebras, American Mathematical Society Colloquium Publications, vol. XXXIX, Providence, R. I., 1968.

Received July 20, 1976.

UNIVERSITY OF WISCONSIN

MADISON, WI 53705 



\section{PACIFIC JOURNAL OF MATHEMATICS}

EDITORS

RICHARD ARENS (Managing Editor)

University of California

Los Angeles, California 90024

C. W. Curtis

University of Oregon

Eugene, OR 97403

C. C. MOORE

J. DUGUNDJI

Department of Mathematics University of Southern Californa Los Angeles, California 90007

R. Finn AND J. Milgram Stanford University Stanford, California 94305

University of California

Berkeley, CA 94720

\section{ASSOCIATE EDITORS}

E. F. BeCKENBACH

B. H. NeUMANN

F. WOLF

K. YosHida

\section{SUPPORTING INSTITUTIONS}

UNIVERSITY OF BRITISH COLUMBIA UNIVERSITY OF SOUTHERN CALIFORNIA CALIFORNIA INSTITUTE OF TECHNOLOGY UNIVERSITY OF CALIFORNIA MONTANA STATE UNIVERSITY UNIVERSITY OF NEVADA, RENO STANFORD UNIVERSITY UNIVERSITY OF TOKYO UNIVERSITY OF UTAH NEW MEXICO STATE UNIVERSITY WASHINGTON STATE UNIVERSITY OREGON STATE UNIVERSITY UNIVERSITY OF WASHINGTON UNIVERSITY OF OREGON OSAKA UNIVERSITY 


\section{Pacific Journal of Mathematics \\ Vol. 73, No. 1 \\ March, 1977}

Thomas Robert Berger, Hall-Higman type theorems. $V \ldots \ldots \ldots \ldots \ldots \ldots \ldots$

Frank Peter Anthony Cass and Billy E. Rhoades, Mercerian theorems via

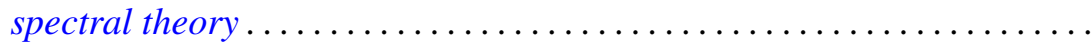

Morris Leroy Eaton and Michael David Perlman, Generating $\mathrm{O}(n)$ with

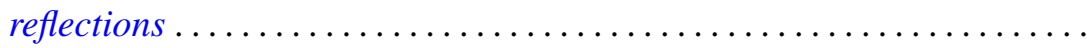

Frank John Forelli, Jr., A necessary condition on the extreme points of a

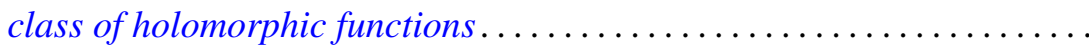

Melvin F. Janowitz, Complemented congruences on complemented

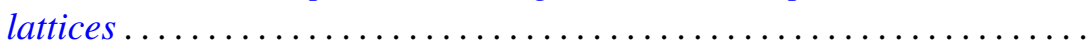

Maria M. Klawe, Semidirect product of semigroups in relation to amenability, cancellation properties, and strong $F \phi$ lner conditions....

Theodore Willis Laetsch, Normal cones, barrier cones, and the "spherical image" of convex surfaces in locally convex spaces ................

Chao-Chu Liang, Involutions fixing codimension two knots.............

Joyce Longman, On generalizations of alternative algebras .............

Giancarlo Mauceri, Square integrable representations and the Fourier

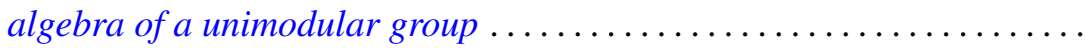

J. Marshall Osborn, Lie algebras with descending chain condition...

John Robert Quine, Jr., Tangent winding numbers and branched

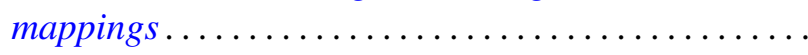

Louis Jackson Ratliff, Jr. and David Eugene Rush, Notes on ideal covers and associated primes .

H. B. Reiter and N. Stavrakas, On the compactness of the hyperspace of faces.

Walter Roth, A general Rudin-Carlson theorem in Banach-spaces ..

Mark Andrew Smith, Products of Banach spaces that are uniformly rotund in every direction.

Roger R. Smith, The R-Borel structure on a Choquet simplex ...

Gerald Stoller, The convergence-preserving rearrangements of real infinite series. ...

Graham H. Toomer, Generalized homotopy excision theorems modulo a Serre class of nilpotent groups...

Norris Freeman Weaver, Dehn's construction and the Poincaré conjecture....

Steven Howard Weintraub, Topological realization of equivariant intersection forms... 